\title{
Die Wiederentdeckung der Anaphylaxie
}

$\mathrm{D}$

as Phänomen ist uralt, auch wenn sich die Historiker noch streiten, ob Pharao Menes im Jahre 2640 v. Chr. durch den Stich einer Wespe oder einen Nilpferdunfall ums Leben kam. Die klinischen Symptome werden in der alten griechischen und chinesischen medizinischen Literatur beschrieben und waren bekannt genug, um Shakespeare im Königsdrama „Richard III“ anzuregen. 1901 wurde es von Richet und Portier wissenschaftlich erkannt und beschrieben und vor allen Dingen „benannt": Die unsterblichen Worte von Charles Richet beim Anblick des im anaphylaktischen

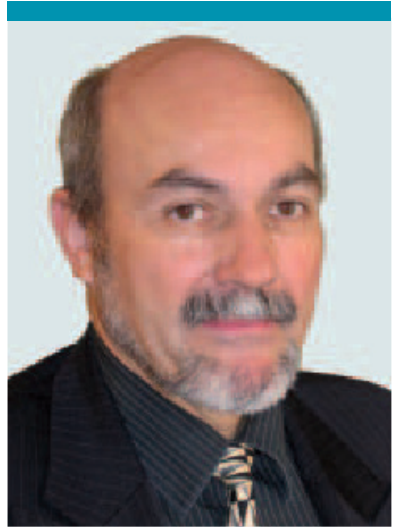

Prof. Dr. Dr. Johannes Ring, Klinik und Poliklinik für Dermatologie und Allergologie am Biederstein, Technische Universität München

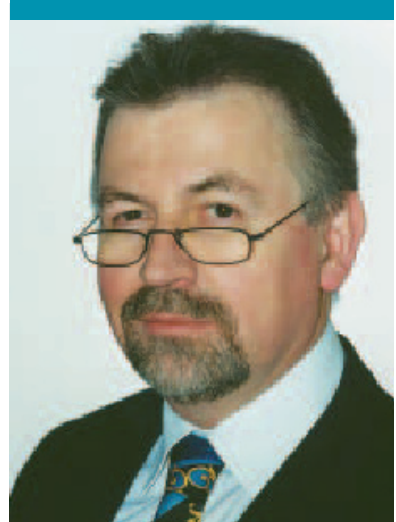

Prof. Dr. Bernhard Przybilla, Klinik und Poliklinik für Dermatologie und Allergologie, Klinikum der Ludwig-Maximilians-Universität, München

der Leitlinie „Akuttherapie anaphylaktischer Reaktionen " erscheint, die in Zusammenarbeit verschiedener Fachgesellschaften und Patientenorganisationen in mehreren Konsensuskonferenzen erarbeitet wurde. Die Leitlinie richtet sich vorwiegend an Ärzte, es ist jedoch unstrittig, dass eine bessere Information für die Betroffenen vordringlich notwendig ist. Deshalb werden zurzeit Programme zur „Anaphylaxieschulung" vorbereitet und hoffentlich ebenso erfolgreich umgesetzt, wie dies für die Asthma- und Neurodermitisschulungen vorbildlich gezeigt werden konnte.

Es handelt sich um eine S2Schock gestorbenen Hundes Neptune zeigen exemplarisch die Wichtigkeit der Terminologie: „C'est un phénomène nouveau, il faut le baptiser"!

In den letzten Jahren scheint die Allergologengemeinde die Anaphylaxie quasi wieder entdeckt zu haben, vielleicht auch wegen steigender Prävalenzzahlen im $\mathrm{Zu}$ - „Es ist letztendlich immer noch sammenhang mit der insgesamt steigenden Anzahl von Allergien. Leider gibt es zur Epidemiologie dieser lebensbe-

und/oder Atemstillstand führen. Deshalb müssen auch leichte Ausprägungen der anaphylaktischen Reaktion ernst genommen werden.

Scheinbar zufällig fügt sich der 30. Geburtstag der Schweregradklassifikation, wie sie 1977 vorgeschlagen wurde (Ring J, Messmer K. Lancet unklar, wie ein paar Es sind seither verschieMikrogramm eines Allergens dene Klassifikationsveres schaffen, einen gesunden suche vorgestellt worden, baumstarken Mann vom Leben alle mit Vor- und Nachzum Tode zu befördern."

teilen, die jedoch sämtlich die Vielfalt und unternur wenig exakte Daten. Glaubwürdige Schätzungen und retrospektive Studien geben Hinweise auf eine Gesamtprävalenz zwischen 1 und 15\% in Abhängigkeit von unterschiedlichen Verlaufsformen in der Allgemeinbevölkerung. Es ist mit einer erheblichen Dunkelziffer zu rechnen.

Gerade in Zeiten, in denen „Allergie“ von nicht Betroffenen oder wenig Informierten - leider auch von gesundheitspolitisch einflussreichen Stellen - bagatellisiert wird als Befindensstörung, muss darauf hingewiesen werden, dass man an Allergien sterben kann. Anaphylaxie ist ein potenziell lebensbedrohliches Ereignis. Was als Urtikaria und Juckreiz an Handflächen und Fußsohlen beginnt, kann innerhalb von Minuten zum Kreislauf- schiedliche Intensität der variablen klinischen Symptome im Gesamtbild der Anaphylaxie betonen. Das ist das Entscheidende.

1994 hatte die Deutsche Gesellschaft für Allergologie und Klinische Immunologie (DGAKI) das Ergebnis einer interdisziplinären Konsensuskonferenz zur Akutbehandlung der Anaphylaxie als Positionspapier im Allergo Journal veröffentlicht (Tryba et al. Allergo J 1994; 3: 211-24). Es ist deshalb besonders erfreulich, dass in diesem Heft die Neufassung

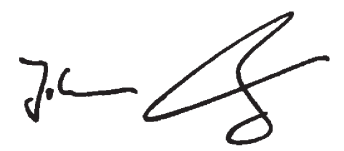

Prof. Dr. Dr. Johannes Ring
Leitlinie, viele hätten sich ein S3-Papier gewünscht. Allerdings ergab die Durchsicht der Literatur, dass der Evidenzgrad auf diesem Gebiet ernüchternd ist. Die Dramatik der Situation verbietet in den meisten Fällen langwierige wissenschaftliche Überlegungen oder den Einschluss in plazebokontrollierte randomisierte klinische Studien. Jeder, der vor solch einem Notfall steht, ist froh, wenn der Patient überlebt! Tatsächlich stellen wir bei Betrachtung der komplexen Pathophysiologie der anaphylaktischen Reaktion fest, wie viel Forschungsbedarf tatsächlich noch besteht. Es ist für den erfahrenen Allergologen immer noch letztendlich unklar, wie ein paar Mikrogramm eines Allergens es schaffen, einen sonst gesunden baumstarken Mann vom Leben zum Tode zu befördern.

Die neue Leitlinie möge nicht nur den Betroffenen zu einer besseren Versorgung helfen, sondern auch junge Forscher anregen - und zwar aus verschiedensten Gebieten, insbesondere auch der Kreislaufphysiologie und der Pharmakologie - das Phänomen der Anaphylaxie wieder verstärkt zu beforschen!

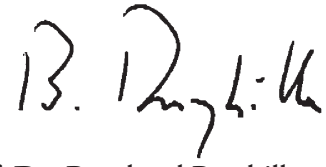

Prof. Dr. Bernhard Przybilla 